\title{
Relations cerne-climat dans des peuplements de Quercus afares Willd et Quercus canariensis Pomel en Algérie
}

\author{
M Messaoudène ${ }^{1}$, L Tessier ${ }^{2 *}$ \\ I INRF, station régionale de Tizi Ouzou, BP 30 Yakouren, 15365, Algérie; \\ ${ }^{2}$ Imep, faculté des sciences de Saint-Jérôme, c 451, 13397 Marseille cedex 20, France
}

(Reçu le 12 février 1996; accepté le 10 juillet 1996)

\begin{abstract}
Summary - Tree-ring to climate relationships for populations of Quercus afares Willd and Quercus canariensis Pomel in Algeria. Ring-width variations are analysed on 15 populations of Quercus afares and Quercus canariensis in the Akfadou and Beni-Ghobri Forests (Algeria) (fig 1) which are submitted to temperate and cool variants of the Mediterranean humid bioclimate. The populations selected are representative of the different ecological conditions (substratum, exposure, altitude) under which the two species are growing. After measuring ring-width on three radii per tree and eight to 16 trees per site, a mean ring-width chronology is developed for each population (table I). In order to remove age trend and isolate interannual variations of radial growth, ring-width series are modelized using ARMA processes. For each population, a mean chronology of residuals is established. Response function is then calculated, involving the mean residual chronology as ciependent variable and, in a first step, the 24 monthly parameters of annual climate as regressors ( $\mathrm{P}-\mathrm{T}_{\text {max }}$ and $\mathrm{P}-\mathrm{T}_{\text {min }}$ ). The number of regressors is then reduced by grouping together the successive monthly values of the same sign. Response function is calculated for the period 1918-1951, where valuable meteorological data are available. The reliability of response function is tested using the bootstrap method. Results obtained from modelization (table II) show that radial growth is largely predetermined for both species. The amount of residual variance to be correlated with climate is low; this amount is lower for $Q$ afares than for $Q$ canariensis. Statistical analysis reveals that neither altitude nor exposure or substratum (table III) can explain the differences between populations. Comparison of the results obtained with response functions (table IV) reveals that, for the two species, precipitation has a greater effect than temperature, the action of which concerns only minimal values. The analysis of global significance of response functions shows that $Q$ afares does not react to climate whereas $Q$ canariensis reacts significantly to the interannual fluctuations of climatic parameters. As the region is submitted to the same regional climate, differences between populations can be attributed to differences in habitats. However, just as with the growth models, it is not possible to establish a significant relationship between the describers of the habitats (altitude, substratum, exposure) and the response functions. In conclusion, the endemic $Q$ afares appears to be in better equilibrium with climate vari-
\end{abstract}

* Correspondance et tirés à part

Tél : (33) 0491288872 ; fax : (33) 0491288668 
ations than $Q$ canariensis; however, as the two species are highly in competition in the same habitat, this equilibrium could be perturbated by a small change in climate.

\section{Algeria / Quercus / ring width / climate / response function / dendroecology}

Résumé - Une analyse des variations de l'épaisseur des cernes en relation avec le climat est menée sur 15 populations de Quercus canariensis et de Quercus afares des massifs forestiers de l'Akfadou et de Beni-Ghobri (Algérie). La palette des situations écologiques (exposition, altitude, substrat) est représentée. La modélisation des séries de largeurs de cernes (modèles Arma) montre que la croissance du cerne des deux espèces est, d'une manière générale, prédéterminée. La part de variance résiduelle mise en jeu dans la relation avec le facteur climatique est moins importante pour $Q$ afares que pour $Q$ canariensis. Aucun des descripteurs écologiques recensés (substrat, exposition, altitude) ne peut isolément expliquer les différences constatées d'une population à l'autre. L'analyse des relations cerne-climat par le calcul des fonctions de réponse montre globalement un rôle dominant des précipitations par rapport aux températures, qui n'interviennent de façon prépondérante que par leurs valeurs minimales. L'intervention de ces paramètres dans un même sens sur le processus de croissance radiale s'étale sur des séquences de 2 à 3 mois. Comme pour la modélisation, les fonctions de réponse révèlent une différence de sensibilité des deux espèces aux fluctuations climatiques, sans qu'aucun des descripteurs écologiques puisse être identifié comme jouant un rôle majeur. En conclusion, $Q$ afares apparaît particulièrement insensible aux fluctuations interannuelles du climat, alors que la croissance de $Q$ canariensis est plus modulée par ces mêmes fluctuations. Cette adaptation de $Q$ afares peut partiellement expliquer le maintien de cette endémique, maintien que tout changement climatique pourrait peut être compromettre.

Algérie / Quercus / cerne / climat / fonction de réponse / dendroécologie

\section{INTRODUCTION}

La chênaie caducifoliée occupe en Algérie une aire de près de 65000 ha. Elle est essentiellement constituée par les deux espèces Quercus canariensis Willd et $Q$ afares Pomel. $Q$ afares est endémique de la région centre-est de l'Algérie; en revanche, l'aire occupée en Algérie par $Q$ canariensis ne constitue qu'une partie de son aire biogéographique (Quezel et Bonin, 1980). Actuellement, le développement de ces chênaies est important, marqué surtout par l'extension de $Q$ canariensis. Si l'extension de cette dernière espèce se fait surtout au détriment de la suberaie, le maintien des peuplements de $Q$ afares dispersés au sein de la chênaie de $Q$ canariensis pourrait poser un problème dans le futur. L'aménagement et la gestion de ces chênaies supposent une bonne connaissance de l'écologie de ces deux essences, en particulier de leur productivité et de leur comportement respectif vis-à-vis du facteur climatique en fonction des biotopes occupés. Une première publication (Messaoudène et Tessier, 1990) traitait de la productivité ; celle-ci présente une analyse comparée des relations cerne-climat pour les mêmes populations représentatives de l'ensemble des forêts de l'Akfadou et BeniGhobri en Kabylie, dont une cartographie avait été établie (Messaoudène, 1989) (fig 1).

\section{MATÉRIEL ET MÉTHODES}

Vingt populations ont été initialement échantillonnées (dix pour $Q$ afares et dix pour $Q$ canariensis), dont quatre populations doubles associant les deux essences. Elles fournissent une représentation aussi exhaustive que possible des différents biotopes occupés dans les deux massifs. La zone géographique couverte appartient au bioclimat méditerranéen humide à variantes fraîche et tempérée et aux étages de végétation des thermo-, méso- et supraméditerranéens (Quezel, 1956 ; Messaoudène et Tessier, 1990). 


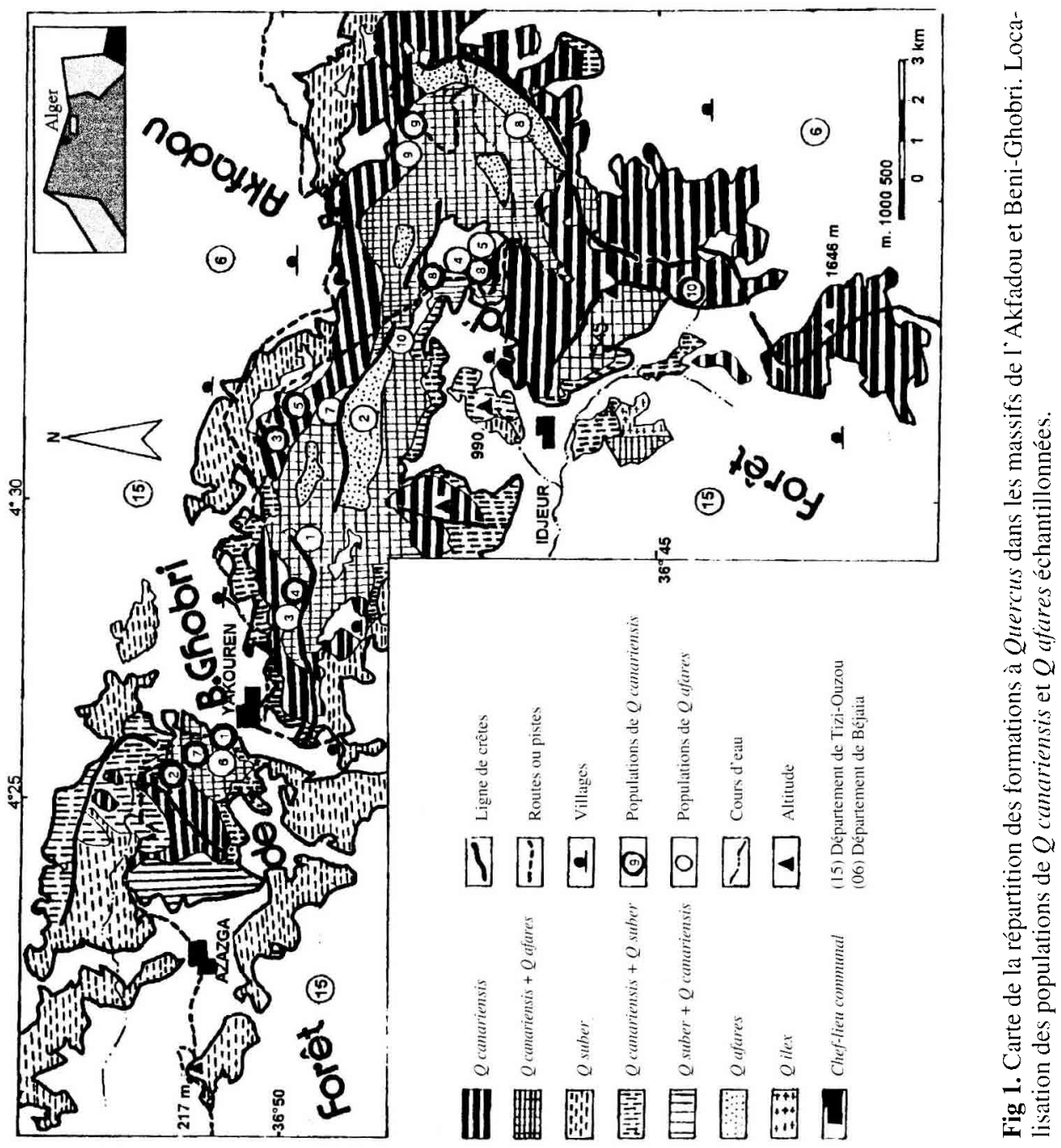

Le choix initial des arbres (huit à seize par site), les sondages à la tarière de Pressler, la préparation des éprouvettes, l'interdatation des séries de cernes puis leur mesure au $1 / 100^{2}$ de millimètre ont été effectués conformément aux critères et aux méthodes établis entre autres par Stokes et Smiley (1968), Fritts (1976) et Cook et Kairiukstis (1990). Au total, sur 243 individus de l'une ou de l'autre espèce, 780 carottes ont été extraites puis traitées. Les chronologies moyennes obtenues (tableau I) couvrent globalement la période maximale 1745-1986.

Pour chaque arbre, l'épaisseur du cerne annuel peut être considérée comme la résultante de l'intervention combinée sur les processus de croissance de son potentiel génétique et des facteurs biotiques et abiotiques associés au biotope qu'il occupe (Fritts, 1976; Serre, 1976 ; Aussenac, 1978 : Becker et Levy, 1986). La séparation des parts de variance de l'épaisseur du cerne 
Tableau I. Caractéristiques des populations et des chronologies de $Q$ canariensis et $Q$ afares. Les populations mixtes sont repérées par le même symbole.

\begin{tabular}{|c|c|c|c|c|c|}
\hline Populations & Code & $\begin{array}{l}\text { Nombre } \\
\text { d'arbres }\end{array}$ & Période & $\begin{array}{c}\hat{A g e} \\
\text { maximal }\end{array}$ & $\begin{array}{c}\hat{A} g e \\
\text { moyen }\end{array}$ \\
\hline \multicolumn{6}{|l|}{ Qafures } \\
\hline Tamadjarth & Qal & 08 & $1866-1985$ & 120 & 116 \\
\hline Tizi. Bousgou & Qa2 & 10 & $1847-1985$ & 139 & 125 \\
\hline Tala Taxirine & Qa3 & 10 & $1863-1985$ & 123 & 114 \\
\hline Tala Kitane 1 & Qa4D & 16 & $1860-1986$ & 127 & 97 \\
\hline Tala Kitane 2 & Qa5 & 11 & $1765-1986$ & 222 & 124 \\
\hline Hôtel Tamgout 2 & Qa6• & 14 & $1871-1986$ & 116 & 110 \\
\hline Tizi Bousgou 2 & Qa7 & 13 & $1855-1986$ & 132 & 124 \\
\hline Borne 11 & Qa8 & 12 & $1790-1986$ & 197 & 150 \\
\hline Agoulmine & Qa9 & 06 & $1890-1986$ & 96 & 90 \\
\hline Tizi El Vaz & Qalo & 07 & $1860-1986$ & 127 & 122 \\
\hline \multicolumn{6}{|l|}{ Q canariensis } \\
\hline Hôtel Tamgout 2 & $\mathrm{Qcl} \bullet$ & 13 & $1870-1986$ & 117 & 105 \\
\hline Font Frầche & Qc2 & 12 & $1863-1986$ & 124 & 104 \\
\hline Limite & $\mathrm{Qc} 3$ & 15 & $1870-1986$ & 117 & 100 \\
\hline Tala Taxirine & Qc4 & 11 & $1867-1986$ & 120 & 105 \\
\hline Font Cor & Qc5 & 15 & $1900-1986$ & 87 & 72 \\
\hline Tala Kitane 3 & Qc6 & 13 & $1800-1986$ & 187 & 139 \\
\hline Hôtel Tamgout 1 & Qc7 & 16 & $1940-1986$ & 47 & 44 \\
\hline Tala Kitane 1 & Qc8 $\square$ & 13 & $1865-1986$ & 122 & 92 \\
\hline Agoulmine & Qc9 & 14 & $1830-1986$ & 157 & 115 \\
\hline Ainseur n'Zéen & Qclo & 13 & $1745-1986$ & 242 & 176 \\
\hline
\end{tabular}

annuel passe par une modélisation des séries chronologiques de cernes. Sont ainsi séparées les variations aléatoires interannuelles et les variations de basse fréquence ou tendancielles.

La modélisation de la croissance sur chaque série d'épaisseurs de cernes fait appcl à la procédure Arma (auto regressive moving average) (Box et Jenkins, 1970) adaptée à l'analyse dendrochronologique (Guiot et al. 1982 ; Tessier, 1987 ; Guiot, 1990). Un modèle calculé sur la base des autocorrélations des séries d'épaisseurs brutes permet d'obtenir l'expression de l'épaisseur du cerne au temps $t\left(C_{t}\right)$ en fonction des valeurs aux temps $t-1, t-2 \ldots . . t-n$. Dans le cas présent, les modèles utilisés ne mettent en jeu au maximum que les 3 années antérieures à l'éla- boration du cerne de l'année t. La partie résiduelle (a) , obtenue par soustraction de la partie modélisée à la largeur mesurée du cerne, correspond aux variations aléatoires, liées à l'action du climat considérée également comme variant aléatoirement d'une année à l'autre. Pour un modèle $\mathrm{AR}_{3} \mathrm{MA}_{1}$, l'un des plus fréquemment utilisés, l'expression mathématique correspondante est la suivante :

$\mathrm{C}_{\mathrm{t}}=\varphi_{1} * \mathrm{C}_{\mathrm{t}-1}+\varphi_{2} * \mathrm{C}_{\mathrm{t}-2}+\varphi_{3} * \mathrm{C}_{\mathrm{t}-3}-\theta_{1} * \mathrm{a}_{\mathrm{t}-1}+\mathrm{a}_{\mathrm{t}}$

Le modèle sélectionné est celui qui fournit des séries de résidus présentant des autocorrélations non significatives (bruit blanc), une variance résiduelle minimale et un $\chi^{2}$ sur les cofficients 
d'autocorrélation de $n-1$ à $n-13$ des résidus minimal. Le modèle unique sélectionné fournit donc, pour chaque série élémentaire d'épaisseurs brutes, une série de résidus et un modèle caractérisé par les valeurs $\varphi$ et $\theta$ comprises entre 0 et 1 . Le modèle représentatif de la population est obtenu en faisant la moyenne arithmétique des coefficients $\varphi$ et $\theta$ des modèles obtenus pour chaque série élémentaire d'épaisseurs brutes. La série correspondante des résidus représentative de la population est obtenue de la même façon en calculant la moyenne des résidus des séries élémentaires précédentes.

L'analyse des relations cerne-climat porte sur 15 populations. Outre les populations fournissant des séries de cernes trop courtes (la période juvénile est en particulier écartée de l'analyse) au regard de la période couverte par les données météorologiques (1918-1951), les populations trop hétérogènes au regard de l'âge des arbres ont été écartées. Le calcul de la relation cerne-climat met en jeu, pour les 15 populations retenues, les séries de résidus précédentes et, initialement, les deux combinaisons (P-Tmax et PTmin) de 24 paramètres climatiques : totaux pluviométriques mensuels et températures moyennes mensuelles (maximale et minimale) du poste de Larbaa-Nath-Irathen (ex-Fort National). Le calcul effectué met en parallèle le résidu de l'année $n$ avec les paramètres climatiques mensuels de la période octobre $n-1$ à septembre de l'année $n$ (Berger et al, 1979 ; Tessier, 1989) correspondant à la séquence 1918-1951. Le caractère discontinu et incomplet des données instrumentales fournies par les postes météorologiques proches de la zone étudiée n'a pas permis d'utiliser les séries les plus récentes. Compte tenu de l'instabilité de la régression, qui met en jeu 24 régresseurs et seulement 34 observations, des regroupements de variables ont été réalisés pour réduire le nombre des régresseurs (Guiot, 1981). Après plusieurs essais, le regroupement utilisé est le suivant : Oct-Nov-Déc, Janv-Févr, Mars-Avr, Mai-Juin-Juill, Août-Sept pour les précipitations et Oct, Nov-Déc, Janv, Févr-Mars-Avr, MaiJuin-Juill et Août-Sept pour les températures maximales et minimales. Ces regroupements sont retenus dans la mesure où ils fournissent les fonctions de réponse les plus significatives sur la majorité des séries élémentaires étudiées. Le nombre de variables explicatives est donc réduit à 11 : soit cinq variables précipitation et six variables température.

Le calcul de la fonction de réponse fait appel à la procédure « Bootstrap » (Efron, 1979 ; Dia- conis et Efron, 1983 ; Guiot, 1990, 1991) ; il met en œuvre une analyse préalable en composantes principales des variables explicatives. Les composantes principales introduites dans la régression sont sélectionnées au seuil PVP (Produit des valeurs propres) supérieur à I (Guiot, 1981). La régression entre variables explicatives et variable dépendante est effectuée 50 fois sur des années observations tirées au sort. La signification globale de la fonction de réponse est fournie par le rapport de la valeur moyenne (pour les 50 tirages au sort) des coefficients de corrélation entre variable réelle et variable estimée du climat calculée à partir des coefficients de régression, sur les années de calibration (années tirées) et les années de vérification (années non tirées). Le rapport de ces coefficients de corrélation aux écarts types respectifs permet d'estimer la fiabilité des résultats. De la même façon, le rapport de la valeur moyenne de chaque coefficient de régression partiel à son écart type (S) établit sa signification. Chaque fonction de réponse est présentée sous une forme codée à partir des coefficients de régression partiels $(\mathrm{R})$ soit : $\mathrm{R}>\mathrm{S}=1$, $\mathrm{R}>2 \mathrm{~S}=2, \mathrm{R}>3 \mathrm{~S}=3, \mathrm{R}>4 \mathrm{~S}=4, \mathrm{R}>5 \mathrm{~S}=5$. Le signe + est attribué aux relations directes et le signe - aux relations de type inverse; seuls les codes supérieurs à 1 sont retenus.

\section{RÉSULTATS}

\section{Les modèles de croissance}

Le tableau II récapitule les résultats obtenus pour l'ensemble des populations. Si les modèles moyens sont de type complexe (22,2-1,1-1) pour certaines populations, une majorité de modèles 1-0 apparaît. Dans le cas des modèles complexes, le coefficient $\varphi_{1}$ qui exprime le poids des autocorrélations d'ordre 1 dans les séries de cernes, est toujours représenté par des valeurs très supérieures.

La part de variance expliquée par les modèles $\left(\mathrm{S}^{2} \mathrm{~m}\right)$ varie de 76 à $33 \%$; celle expliquée complémentairement par les résidus $\left(\mathrm{S}^{2} \mathrm{e}\right)$ de 67 à $24 \%$. Les différences, d'une population à l'autre, les deux espèces confondues, sont importantes, mais les valeurs sont significativement différentes 
Tableau II. Modèles de croissance représentatifs des populations de $Q$ canariensis et de $Q$ afares.

\begin{tabular}{|c|c|c|c|c|c|c|c|c|c|}
\hline \multirow[b]{2}{*}{$P O P$} & \multirow[b]{2}{*}{$M O D$} & \multicolumn{5}{|c|}{ Coefficients des modeles } & \multicolumn{3}{|c|}{ Efficacité de la modélisation } \\
\hline & & $\varphi_{1}$ & $\varphi_{2}$ & $\varphi_{3}$ & $\theta_{1}$ & $\theta_{2}$ & $\% S^{2} e$ & $\% S^{2} m$ & $\chi^{2}$ \\
\hline \multicolumn{10}{|c|}{$Q$ aftares } \\
\hline 1 & 1,1 & 0,78 & & & 0,15 & & 24 & 76 & 11,9 \\
\hline 2 & 2,0 & 0,57 & 0,15 & & & & 40 & 60 & 11,4 \\
\hline 3 & 1,0 & 0,54 & & & & & 58 & 42 & 9,5 \\
\hline 4 & 1,0 & 0,70 & & & & & 42 & 58 & 11,5 \\
\hline 5 & 2,2 & 0,80 & $-0,22$ & & 0.25 & 0,15 & 48 & 52 & 8,4 \\
\hline 6 & 1,0 & 0,50 & & & & & 61 & 39 & 9,1 \\
\hline 7 & 1,0 & 0,59 & & & & & 62 & 38 & 10,2 \\
\hline 8 & 2,0 & 0,63 & 0,22 & & & & 35 & 65 & 11,4 \\
\hline 9 & 1,1 & 0,78 & & & 0,25 & & 50 & 50 & 10,2 \\
\hline 10 & 1,0 & 0,65 & & & & & 56 & 44 & 10,2 \\
\hline \multicolumn{10}{|c|}{$Q$ canariensis } \\
\hline 1 & 2,1 & 0.83 & 0,21 & & 0,28 & & 57 & 43 & 7,8 \\
\hline 2 & 1,0 & 0,65 & & & & & 61 & 39 & 7,7 \\
\hline 3 & 2,0 & 0,49 & 0,17 & & & & 45 & 55 & 10,0 \\
\hline 4 & 1,0 & 0,55 & & & & & 53 & 47 & 8,1 \\
\hline 5 & 1,0 & 0.80 & & & & & 38 & 62 & 7,2 \\
\hline 6 & 3,0 & 0,62 & $-0,24$ & 0,19 & & & 49 & 51 & 8,7 \\
\hline 8 & 1,1 & 0,86 & & & 0,22 & & 52 & 48 & 10,8 \\
\hline 9 & 1,1 & 0,59 & & & 0,17 & & 65 & 35 & 8,8 \\
\hline 10 & 1,0 & 0,50 & & & & & 67 & 33 & 7,5 \\
\hline
\end{tabular}

Pop : population; Mod : modèle ; \% $S^{2}$ e : pourcentage de variance expliqué par les résidus ; $\% S^{2} \mathrm{~m}$ : pourcentage de variance expliqué par le modèle $; \chi^{2}$ : Chi carré sur les coefficients d'autocorrélation des résidus ( $n-1$ à $n-13)$.

d'une population à l'autre : le test $U$ de Mann et Whitney appliqué aux deux séries de valeurs montre que l'identité des deux distributions est rejetée au seuil $0,5 \%$. Lorsque les deux espèces cohabitent sur le même site, les modèles identifiés pour les deux populations correspondantes sont différents dans deux cas sur quatre. Dans un des deux cas où le même modèle est identifié, les pourcentages de variance expliquée différencient fortement les deux espèces. Seules les populations mixtes Qa3 et Qc4 sont caractérisées par des modèles presque identiques. Sur la base de l'échantillon retenu, chaque espèce a donc des caractéristiques de croissance, représentées par le modèle identifié, qui lui sont propres.

Parmi les données écologiques répertoriées (Messaoudène et Tessier, 1991), celles qui paraissaient les plus discriminantes ont été testées : soit, le substrat (argileux ou exclusivement gréseux), l'altitude (>1000 m ou < $1000 \mathrm{~m}$ ), l'exposition ( $\mathrm{N}$ ou $\mathrm{W}$ et $\mathrm{S}-\mathrm{W}$ ) (tableau III). Le même test de rang, appliqué dans ces trois cas aux deux groupes de valeurs correspondantes $\left(S^{2} M\right)$, ne révèle pas de différences significatives. Les différences constatées d'une population 
Tableau III. Caractéristiques écologiques des stations correspondant aux différentes populations..

\begin{tabular}{ccccc}
\hline Populations & Expositions & Substrats & Altitude & Pente (\%) \\
\hline Q afares & & & & \\
1 & SW & Grès et argile & 1110 & 15 \\
2 & SW & Argile & 1150 & 20 \\
3 & $\mathrm{~N}$ & Grès & 950 & 30 \\
4 & $\mathrm{~N}$ & Grès & 1150 & $12-25$ \\
5 & $\mathrm{SW}$ & Argile & 1160 & 15 \\
6 & $\mathrm{~N}$ & Grès & 780 & $20-30$ \\
8 & $\mathrm{~W}$ & Grès & 1350 & 2 \\
9 & $\mathrm{~N}$ & Grès & 1280 & 20 \\
& & & & \\
Qcanariensis & & Grès & 780 & $20-30$ \\
1 & $\mathrm{~N}$ & Grès +argile & 740 & $25-50$ \\
2 & $\mathrm{~N}$ & Grès +argile & 920 & 20 \\
3 & $\mathrm{~N}$ & Grès & 1100 & $5-10$ \\
6 & $\mathrm{~W}$ & Grès & 1150 & $12-25$ \\
8 & $\mathrm{~N}$ & Grès & 1280 & 20 \\
9 & $\mathrm{~N}$ & Grès & 1400 & $12-25$ \\
10 & $\mathrm{~W}$ & & & \\
\hline
\end{tabular}

à l'autre, donc d'un biotope à l'autre, résultent ainsi de la combinaison des facteurs stationnels sans qu'il soit possible d'isoler un de ces facteurs. Par ailleurs, sur la base de l'échantillon traité, aucun de ces facteurs écologiques ne semble préférentiellement être attaché à l'une ou à l'autre espèce.

\section{Les fonctions de réponse}

Le tableau IV regroupe les résultats obtenus sur les populations de l'une et l'autre espèce. Si la signification globale de la fonction de réponse (seuil $99 \%$ ) est évidente sur les années de calibration, en revanche cette signification est relativement plus faible sur les années de vérification. La différence traduit l'instabilité temporelle de la relation cerne-climat.

La confrontation des significations globales obtenues pour les deux combinaisons de paramètres climatiques ( $\mathrm{P}-\mathrm{Tm}$ min et $\mathrm{P}$ Tmax) fait ressortir un rôle systématique- ment plus important des températures minimales dans le déterminisme de la croissance. Pour le couple P-Tmax, la fonction de réponse n'est globalement significative au seuil de $95 \%$ que pour les seules populations Qa6 et Qc1, Qc8. On notera que Qa6 et Qcl croissent en mélange dans le même biotope. L'action des températures ne sera donc prise en compte ultérieurement qu'au niveau des températures minimales.

La relation cerne-climat n'est globalement significative au seuil minimal $90 \%$ que pour quatre des huit populations de $Q$ afares et six des sept populations de $Q$ canariensis. Cependant, l'examen du tableau IV montre que, pour les populations caractérisées par des fonctions de réponse globalement non significatives, les paramètres identifiés comme intervenant significativement (code $2 \mathrm{ou}>2$ ) sont les mêmes, et surtout qu'ils interviennent le plus souvent, quelle que soit l'espèce, dans le même sens (direct ou inverse). Il y a donc cohérence globale 
Tableau IV. Fonctions de réponse des différentes populations des deux chênes.

\begin{tabular}{|c|c|c|c|c|c|c|c|c|c|c|c|c|c|c|c|c|}
\hline \multirow[t]{3}{*}{$N^{\circ}$} & \multirow[b]{3}{*}{$C$} & \multirow{3}{*}{\multicolumn{2}{|c|}{$R M C R M V$}} & \multirow[b]{3}{*}{$S C$} & \multirow{3}{*}{ SV } & \multirow{3}{*}{$\begin{array}{c}\text { Seuil } \\
\text { de sign } \\
\text { global } \\
(\%) \\
\text { RMC }\end{array}$} & \multirow{3}{*}{$\begin{array}{c}\text { Seuil } \\
\text { de sign } \\
\text { global } \\
(\%) \\
\text { RMV }\end{array}$} & \multicolumn{9}{|c|}{ Régresseurs } \\
\hline & & & & & & & & \multicolumn{4}{|c|}{ Précipitations } & \multicolumn{5}{|c|}{ Températures } \\
\hline & & & & & & & & $O N D J F$ & $M A$ & $M J J$ & $A S$ & $O N D$ & $J I$ & $F M A$ & $M J J$ & $A S$ \\
\hline \multicolumn{17}{|c|}{$Q$ afares } \\
\hline \multirow[t]{2}{*}{1} & 1 & 79 & 34 & 0,06 & 0,19 & 99 & 90 & & -2 & 2 & & & -2 & -2 & -3 & 2 \\
\hline & 2 & 76 & 27 & 0,09 & 0,32 & 99 & & -2 & -2 & 3 & & & & & & \\
\hline \multirow[t]{2}{*}{2} & 1 & 80 & 33 & 0,07 & 0,20 & 99 & 90 & -3 & & 2 & & & & & -3 & \\
\hline & 2 & 77 & 27 & 0,09 & 0,23 & 99 & 70 & -3 & & 3 & & & & & & \\
\hline \multirow[t]{2}{*}{3} & 1 & 74 & 21 & 0,07 & 0,20 & 99 & 70 & & & & & & & & -3 & \\
\hline & 2 & 65 & 40 & 0,01 & 0,23 & 99 & & & & & & & & & & \\
\hline \multirow[t]{2}{*}{4} & 1 & 78 & 28 & 0,09 & 0,23 & 99 & 70 & -2 & -2 & & & & & & & \\
\hline & 2 & 74 & 15 & 0,07 & 0,22 & 99 & & -3 & -2 & 2 & & & & & & \\
\hline \multirow[t]{2}{*}{5} & 1 & 84 & 53 & 0,06 & 0,21 & 99 & 95 & -2 & -2 & 2 & -2 & $2-2$ & & & -2 & \\
\hline & 2 & 75 & 24 & 0,07 & 0,21 & 99 & 70 & -3 & -2 & 2 & & & & & -2 & \\
\hline \multirow[t]{2}{*}{6} & 1 & 82 & 61 & 0,05 & 0,18 & 99 & 99 & & & & & 2 & & & -4 & \\
\hline & 2 & 80 & 46 & 0,06 & 0,20 & 99 & 95 & & & 2 & & 2 & 2 & 2 & -3 & \\
\hline \multirow[t]{2}{*}{8} & 1 & 70 & 23 & 0,07 & 0,27 & 99 & & -4 & -2 & & -2 & 2 & & & & \\
\hline & 2 & 69 & 25 & 0,08 & 0,21 & 99 & 70 & -5 & -2 & & & & & & & \\
\hline \multirow[t]{2}{*}{9} & 1 & 68 & 5 & 0,10 & 0,23 & 99 & & & -2 & & & & & & -2 & \\
\hline & 2 & 63 & 7 & 0,08 & 0,23 & 99 & & & -2 & & & & & & & \\
\hline
\end{tabular}

\section{$Q$ canariensis}

\begin{tabular}{|c|c|c|c|c|c|c|c|c|c|c|c|c|c|}
\hline \multirow[t]{2}{*}{1} & 86 & 60 & 0,03 & 0,15 & 99 & \multicolumn{2}{|l|}{99} & \multirow{2}{*}{$\begin{array}{l}-2 \\
-2\end{array}$} & 2 & \multicolumn{3}{|c|}{-2} & -3 \\
\hline & 81 & 51 & 0,07 & 0,19 & 99 & 99 & & & 2 & -2 & 2 & & -2 \\
\hline \multirow[t]{2}{*}{2} & 80 & 41 & 0,05 & 0,22 & 99 & 99 & & & 2 & & -2 & & -2 \\
\hline & 78 & 29 & 0,08 & 0,27 & 99 & 70 & & & 2 & & -22 & 2 & -2 \\
\hline \multirow[t]{2}{*}{3} & 78 & 37 & 0,06 & 0,25 & 99 & 70 & & & & & & & -3 \\
\hline & 69 & 15 & 0,08 & 0,27 & 99 & & & & & & & & \\
\hline & 80 & 38 & 0,06 & 0,24 & 99 & 90 & -2 & -2 & & & & & -2 \\
\hline & 77 & 30 & 0,06 & 0,21 & 99 & 70 & -2 & -3 & 2 & & & & -2 \\
\hline & 83 & 50 & 0,05 & 0,17 & 99 & 99 & -2 & & & 2 & & & -2 \\
\hline & 79 & 39 & 0,05 & 0,20 & 99 & 95 & -3 & -2 & 2 & & & & \\
\hline & 78 & 38 & 0,06 & 0,23 & 99 & 90 & -2 & & & 2 & $-2-2$ & -2 & -2 \\
\hline & 71 & 23 & 0,07 & 0,19 & 99 & 70 & -3 & -2 & & & 2 & 2 & \\
\hline \multirow[t]{2}{*}{10} & 82 & 46 & 0,05 & 0,21 & 99 & 90 & & -2 & 2 & 2 & -3 & & -3 \\
\hline & 71 & 17 & 0,07 & 0,21 & 99 & & & -2 & 2 & & & & \\
\hline
\end{tabular}

POP : population ; $\mathrm{C}$ : couple $; 1: \mathrm{P}+\mathrm{Tmin} ; 2: \mathrm{P}+\mathrm{Tmax} ; \mathrm{RMC}$ et RMV : coefficients de corrélation moyen entre les valeurs mesurées et les valeurs estimées du modèle calculés sur les données de calibration (C) et de vérification (V); SC et SV : écarts types des coefficients précédents ; Seuil de sign global : seuil de signification global sur les années de calibration. 
des résultats pour l'ensemble des populations.

L'analyse du profil des fonctions de réponse obtenues à partir des regroupements de variables mensuelles montre que la croissance radiale est essentiellement modulée par le facteur climatique sur des séquences de 2 à 3 mois.

Deux séquences semblent jouer un rôle majeur pour presque toutes les populations : pour les précipitations et la température, la séquence qui va de mars à juillet, et pour les précipitations surtout, la séquence octobre-novembre-décembre. Les autres séquences n'interviennent que pour quelques populations. Très globalement, les précipitations jouent un rôle plus important que les températures pour l'une et l'autre espèce. Les températures interviennent un peu plus nettement sur les populations de $Q$ canariensis, mais, ici aussi, il n'est pas possible de faire la part de ce qui est attribuable au biotope ou à l'espèce.

\section{Relations avec les précipitations}

L'intervention des précipitations peut être décomposée en deux séquences : d'octobre à avril et de mai à juillet. La seconde coïncide avec la phase de construction du cerne.

Dans le début (O N D) et la fin (M A) de la première séquence, les précipitations jouent un rôle favorable sur la croissance lorsqu'elles sont inférieures à la moyenne ; pendant la phase centrale ( $\mathrm{J}$ F), les arbres sont indifférents au facteur précipitation.

Pendant la séquence suivante ( $\mathrm{M} \mathrm{J} \mathrm{J}$ ), des précipitations abondantes interviennent au contraire favorablement sur la croissance radiale.

La cohérence des résultats d'une population à l'autre n'est évidente que dans la mesure où, pour une même séquence, lorsqu'une relation significative intervient, elle est toujours de même type : directe ou inverse. Lorsqu'on tente d'analyser ces résultats (tableau IV) en fonction d'un des critères écologiques répertoriés (tableau III), il n'est guère possible que de dégager quelques tendances.

Ainsi, pour la séquence $\mathrm{OND}$, les populations caractérisées par une relation inverse avec les précipitations au cours de cette séquence sont toutes des populations d'altitude. Mais on trouve parmi les populations indifférentes à ce paramètre des populations également d'altitude (Qc 10, Qa8). Ces deux dernières populations sont caractérisées par des sols très compacts de type hydromorphes. Pour les autres séquences, les exceptions à la règle comme la règle ellemême ne peuvent être rattachées à aucun facteur écologique précis.

Pour la séquence $\mathrm{M} A$, la relation inverse affecte également, à une exception près (Qc1), préférentiellement les populations d'altitude.

Sur la séquence $\mathbf{M} \mathbf{J} \mathbf{J}$, des relations précipitation-croissance de type direct affectent, à une exception près, toutes les populations pour lesquelles la fonction de réponse est globalement significative. En fait, cette séquence doit être interprétée en connexion avec la relation inverse mise en évidence avec le régresseur température couvrant la même séquence. Ce couplage précipitation-température est toujours effectif, il concerne préférentiellement les températures minimales et peut être considéré comme un effet « bilan hydrique ».

\section{Relations avec les températures}

La seule relation assez générale sur l'ensemble des populations concerne la séquence $\mathrm{MJ} J$, pour laquelle sont mises en évidence des relations de type inverse. Ces relations sont couplées aux relations directes avec les précipitations qui caractérisent également un maximum de populations de l'une ou l'autre espèce. Les seules populations qui font exception sont celles pour lesquelles 
la fonction de réponse est globalement non significative.

Pour toutes les autres séquences, les relations mises en évidence n'affectent que quelques populations de l'une ou l'autre espèce. La confrontation de ces résultats avec les données écologiques ne fournit que quelques indications plus ou moins cohérentes :

- ainsi, si la relation directe avec les températures d'octobre caractérise les trois populations de plus haute altitude de $Q$ canariensis, elle s'applique à un ensemble hétérogène vis-à-vis de l'altitude de trois populations de $Q$ afares;

- pour les autres séquences (FMA et $\mathbf{J}$ ), les résultats sont encore plus incohérents puisque, selon les populations, apparaissent des relations tantôt directes, tantôt inverses.

\section{DISCUSSION ET CONCLUSION}

Les modèles descripteurs de l'évolution de la croissance radiale analysée dans ses variations interannuelles mettent en évidence un comportement singulier pour l'une et l'autre essence. Pour $Q$ afares, une plus large part de variance $(52 \%$ en moyenne contre $46 \%$ pour $Q$ canariensis) doit être attribuée au modèle de croissance. Une large part de l'épaisseur du cerne est donc prédéterminée avant même que n'interviennent les aléas du climat. La part de variance résiduelle, mise en jeu ultérieurement dans le calcul des fonctions de réponse, est donc un peu plus réduite pour $Q$ afares que pour $Q$ canariensis. L'étude précédente sur les rythmes d'accroissement radial (Messaoudène et Tessier, 1991) montrait que les seules variations interannuelles de l'épaisseur du cerne, exprimées par le coefficient de sensibilité moyenne (SM) (Fritts, 1976), étaient plus fortes dans le cas de $Q$ canariensis $(\mathrm{SM}=20)$ que dans celui de $Q$ afares ( $\mathrm{SM}=17)$. On pourrait donc penser que cette dernière espèce jouit d'une meilleure adaptation au climat régional. Cependant, la même étude montrait une dynamique de croissance supérieure pour $Q$ canariensis, qui se matérialise par un accroissement annuel moyen toujours supérieur à celui de $Q$ afares, et une plus grande sensibilité de $Q$ afares aux phénomènes de compétition, sensibilité matérialisée par les changements dans les rythmes de croissance associés aux interventions sylvicoles.

L'ensemble des fonctions de réponse obtenues met en évidence une grande diversité de comportement vis-à-vis du facteur climatique des différentes populations des deux chênes. Si le couplage des paramètres précipitation et température (relation directe avec les précipitations et inverse avec les températures) est indubitablement lié aux mécanismes écophysiologiques de la croissance pendant la séquence de plein fonctionnement cambial (MJJ), en revanche, sur les autres séquences, ce couplage est moins évident, et les précipitations en particulier interviennent surtout au niveau du stockage de l'eau dans le sol qui conditionne à la fois le développement racinaire et la mobilisation ultérieure de ces réserves en eau (Bonneau et Souchier, 1978 ; Callot, 1982 ; Becker et Levy, 1986). Bien que l'aire analysée soit d'une superficie restreinte, et donc globalement soumise à un même type de climat régional, les populations réagissent différemment. La relation cerne-climat dépend donc pour une large part du biotope. Cependant, si altitude et hydromorphie du sol semblent jouer un rôle, il n'est pas possible d'établir une correspondance statistiquement satisfaisante entre un de ces descripteurs du biotope et un des types de relations cerne-climat mises en évidence par les fonctions de réponse. En revanche, le niveau de signification des fonctions de réponse singularise chaque espèce. Les fonctions de réponse sont davantage significatives dans le cas de $Q$ canariensis. Deux populations seulement de $Q$ afares sont caractérisées par des fonctions de réponse significatives au seuil $95 \%$. Quatre d'entre elles sont carac- 
térisées par des fonctions de réponse non significatives au seuil $70 \%$. Dans le cas de $Q$ canariensis, trois fonctions de réponse sont significatives au seuil $99 \%$. Ces résultats viennent conforter l'interprétation précédemment faite sur la base de l'analyse de la variance attribuable aux modèles de croissance. $Q$ afares est, dans la plupart des situations, indifférent aux variations interannuelles des paramètres du climat ; ce n'est au contraire qu'exceptionnellement (une seule fonction de réponse non significative au seuil $90 \%$ ) que $Q$ canariensis's'affranchit totalement de ces mêmes aléas. Par ailleurs, l'analyse des relations cerne-climat menée sur d'autres populations de $Q$ canariensis du Maghreb (Raouane, 1985 ; Aloui et Serre-Bachet, 1987) montre que, si leur comportement est semblable pour la période estivale (M J J), il devient plus variable pour les autres périodes. Cette variabilité a été rapportée à la différenciation climatique et édaphique des biotopes sur l'ensemble du Maghreb (Tessier et al, 1994).

\section{CONCLUSION}

Les remarques précédentes tendraient à montrer que $Q$ afares bénéficie dans ces sites de conditions de croissance optimales susceptibles de lui conférer un avantage sur $Q$ canariensis. Cet avantage reste cependant ténu, dans la mesure où une faible dérive du climat pourrait modifier l'équilibre entre les deux essences qui cohabitent dans des biotopes a priori identiques, et dans la mesure aussi où les peuplements de l'endémique $Q$ afares se trouvent noyés au sein de la vaste aire de $Q$ canariensis. Si les potentialités de croissance radiale sont un élément important pour le forestier, elles ne permettent pas d'en extrapoler la dynamique de l'essence dans le peuplement, qui dépend aussi et surtout des stratégies de reproduction et d'expansion. Les différentes réactions aux facteurs susceptibles d'intervenir dans le maintien d'une essence endémique comme $Q$ afares ne doivent être considérées que comme des composantes de la stratégie globale de l'espèce pour maintenir ses populations face aux contraintes du milieu (Merbouche, 1996). L'analyse dendroécologique ne fournit des éléments de connaissance que sur la compétitivité de l'essence au stade adulte. Cette compétitivité dépend largement de l'évolution spatiotemporelle des habitats, elle-même déterminée par les perturbations biotiques et abiotiques; elle est donc elle-même évolutive. La prise en compte de ce caractère évolutif, qui pourrait être faite en confrontant l'évolution spatiotemporelle des fonctions de réponse (Tessier, 1989) à des approches de type analyse de la dynamique des peuplements (recrutement, mortalité), se heurte malheureusement aux limitations de disponibilité de séries météorologiques instrumentales fiables et suffisamment longues.

\section{RÉFÉRENCES}

Aloui A. Serre-Bachet F (1987) Analyse dendroclimatologique comparée de six populations de chêne zéen et d'une population de pin maritime du nordouest de la Tunisie. Écol Méditerr 13, 53-73

Aussenac G (1978) La sécheresse de 1976 : influence des déficits hydriques sur la croissance ligneuse. Rev For Fr 30, 103-104

Becker M, Levy G (1986) Croissance radiale comparée de chênes adultes ( $Q$ robur $L$ et $Q$ petraea Matt Liebl) sur sol hydromorphe acide : effet du drainage. Acta Ecol Plant, 121-356

Berger A, Guiot J, Mathieu L, Munaut AV (1979) Treerings and climate in Morocco. Tree-Ring Bull 39, $61-75$

Bonneau M, Souchier B (1978) Pédologie : constituants ef propriétés du sol. tome 2. Masson, Paris

Box GFP, Jenkins GM (1970) Time Series Analysis: Forecasting and Control. Holden Day, San Francisco, USA

Callot G (1982) Les Interactions sol-racines : incidence sur la nutrition minérale. Inra, Paris

Cook ER, Kairiukstis A (1990) Methods of Dendrochronology. Applications in the Environment Sciences. Kluwcr Academic Pub

Diaconis R, Efron B (1983) Méthodes de calculs statistiques intensives sur ordinateurs. Pour la science (juillet 83) 46-58 
Efron B (1979) Bootstrap methods: another look at the Jacknife. Annals Statistics 7, 1-26

Fritts HC (1976) Tree-Ring and Climate. Academic Press, Londres

Guiot J (1981) Analyse mathématique de donnécs géophysiques. Application à la dendroclimatologie. Vol 1. Thèse de doctorat ès sciences, université catholique de Louvain, faculté des sciences, Louvain-la-Neuve

Guiot J (1990) Methods and programs of statistics for paleoclimatology and paleoecology. In: Guiot J, Labeyrie L, eds. With a diskette for PC programs package (3Pbase), J Guiot et C Goeury, Imep, Marscille

Guiot J (1991) The bootstrapped response function. Research report. Tree-Ring Bull 51, 39-41

Guiot J, Tessier L, Serre-Bachet F (1982) Application de la modélisation Arma en dendroclimatologic. CR Acad Sci 294, 133-136

Merbouche M (1996) Structure et dynamique des peuplements de chênes caducifoliés ( $Q$ afares Pomel et $Q$ faginea Willd) dans le massif forestier d'Akfadou (Algérie). Thèse de Magister de l'université Mouloud-Mammeri, Inst Bio Sci Nat, Algérie

Messaoudène M (1989) Dendroécologie et productivité de $Q$ canariensis Willd et de $Q$ afares Pomel dans les massifs de l'Akfadou et de Beni-Ghobri en Algérie. Thèse de docteur en sciences, universitć d'Aix-Marseille III

Messaoudène M, Tessier L (1991) Croissance radiale de $Q$ canariensis Willd et de $Q$ afares Pomel en Kabylie (Algérie). Écol Méditerr, 17, 119-133
Quczel P (1956) Contribution à l'étude des forêts de chênes à feuilles caduques d'Algérie. Mém Soc Hist Nat de I'Afrique du Nord, nouvelle série 1

Quezel P, Bonin G (1980) Les forêts feuillues du pourtour méditerranéen, constitution, écologie, situation actuelle et perspectives. Rev Forest Fr 32, 253 265

Raouane M (1985) Étude dendroclimatologique du chêne zéen (Quercus canariensis Willd) du Rif occidental et du Moyen-Atlas au Maroc. Thèse de docteur troisième cycle, faculté de Saint-Jérôme, université d'Aix-Marseille-III

Serre F (1976) Les rapports de la croissance et du climat chez le pin d'Alep. Méthodes utilisćes : l'activité cambiale et le climal. Acta Oecol Plant 11,143 171

Stokes MT, Smiley TL (1968) An Introduction to TreeRing Dating. The University of Chicago Press

Tessier L (1987) Dendroclimatological approach to the ecology of Quercus pubescens Willd and Pinus silvestris $\mathrm{L}$ in the French Mediterranean area (methodological aspects). In: Proceedings of the International Symposium on ecological aspects of tree-ring analysis. In $\mathrm{CO}_{2}$, April 1987, 599-610

Tessicr L (1989) Spatio-temporal analysis of climate tree-ring relationships. New Phytol 1 1, 517-529

Tessier L, Nola P, Serre-Bachet F (1994) Deciduous Quercus in the Mediterranean region: tree-ring/climate relationships. New Phytol 126, 355-367 